\title{
Lineamientos Metodológicos para construir Ambientes de Aprendizaje en Sistemas Productivos Agropecuarios soportados en Dinámica de Sistemas
}

\author{
Urbano E. Gómez ${ }^{(1)}$, Hugo H. Andrade ${ }^{(2)}$, Carlos A. Vásquez ${ }^{(3)}$ \\ (1) Facultad de Ingeniería de Sistemas e Informática, Universidad Pontificia Bolivariana (UPB), Autopista a \\ Piedecuesta Kilómetro 7, Edif. I, Oficina 301, Bucaramanga-Colombia (e-mail: urbano.gomez@upb.edu.co) \\ (2) Escuela de Ingeniería de Sistemas, Universidad Industrial de Santander (UIS), Calle 9 con Cra 27, Grupo \\ SIMON, Edificio LP, Oficina 316, Bucaramanga-Colombia (e-mail: handrade@uis.edu.co) \\ (3) Instituto de Proyección Regional y Educación a Distancia (IPRED)), Universidad Industrial de Santander \\ (UIS), Edificio IREDI, Oficina 303. Bucaramanga-Colombia (e-mail: cvasquez@uis.edu.co)
}

Recibido Nov. 25, 2014; Aceptado Ene. 15, 2015; Versión final Abr. 2, 2015, Publicado Ago. 2015

\begin{abstract}
Resumen
Este artículo presenta una propuesta metodológica que orienta, facilita y motiva la construcción de ambientes de aprendizaje para la formación y entrenamiento sobre la toma de decisiones, la administración y la investigación de procesos agropecuarios. La metodología está apoyada en modelos de simulación que permiten, mediante matemática no lineal y computación soportada en la Dinámica de Sistemas, la generación de posibles escenarios para abordar la complejidad dinámica de los fenómenos en estudio. La propuesta resulta ser útil y conveniente para aprendices e investigadores que en grupos interdisciplinarios asumen el reto de abordar la complejidad de sistemas productivos.
\end{abstract} simulación.

\section{Methodological Guidelines for Constructing Learning Environments on Animal Husbandry Production Systems supported on System Dynamics}

\begin{abstract}
This paper presents a methodological proposal to motivate and facilitate the construction of learning environments for the formation and training about decision-making, management and research of agriculture and livestock processes. The methodology is supported by simulation models which, by using non-linear mathematics and computing supported by System Dynamics, allow the generation of different scenarios for approaching, the dynamic complexity of the phenomena under study. The proposal is useful and convenient for non-expert and researchers that in interdisciplinary groups decide to study the complexity of real productive systems.
\end{abstract}

Keywords: agriculture and livestock, information technology, system dynamics, modeling and simulation. 


\section{INTRODUCCIÓN}

La complejidad dinámico-sistémica de los fenómenos agropecuarios merece lineamientos metodológicos para facilitar la construcción de ambientes informáticos asumidos como "instrumentos de mediación que posibilitan las interacciones entre los sujetos y median la relación de éstos con el conocimiento, con el mundo, con los hombres y consigo mismo" (Ospina-Pineda, 2013). La Dinámica de Sistemas (DS) es una alternativa rigurosa y flexible (Andrade et al., 2001) para dar cuenta de la dinámica de los sistemas de producción agropecuaria en términos de modelos matemáticos de simulación y la generación de escenarios como elementos de juicio para posibles cambios en función de las variables de decisión. La DS es útil para aprendices, docentes, investigadores y expertos en las actividades de aprendizaje, toma de decisiones, investigación y en la transferencia de tecnología. Este artículo presenta lineamientos que buscan motivar y orientar la construcción de modelos matemáticos integrados en ambientes software para la experimentación simulada de sistemas del sector agropecuario como el bovino, porcino, caprino y cunícola.

La DS hace presencia en el estudio de los sistemas pecuarios de diversas maneras y temáticas. Tal es el caso de experiencias de uso de la DS en el Centro de Bovinos de Leche en el Instituto de Zootecnia Nova Odessa en Brasil (Toledo et al, 2011), las cuales les ha permitido afirmar que "el proceso de construcción de modelos conceptuales con DS contribuye a la construcción de un consenso entre los investigadores y que el modelo conceptual puede ser utilizado para la construcción de modelos computacionales que constituyen herramientas auxiliares para la investigación ganadera". Otras evidencias de modelos para apoyar la toma de decisiones en sistemas productivos agroindustriales han sido presentada por varios autores. Gómez et al., (2010) y Gómez et al., (2003) con SIPROB 2.0 y SIPROB 1.0, describen modelos de ganadería bovina de carácter poblacional construidos con DS enfocados al aprendizaje, la comprensión, la explicación, la intervención y a la experimentación con diferentes escenarios. Los modelos contemplan subsistemas etarios, biofísicos, financieros y productivos. Díaz (2008), describe un modelo de explotación bovina con programación lineal para el análisis y la toma de decisiones en lo económico y ambiental en materia de fertilización, integrando criterios de competitividad, sostenibilidad y equidad, fue llevado a cabo. Mas tarde Lellis et al. (2011) presentan un modelo en el lenguaje de las influencias para dar a conocer la complejidad del sistema de producción lechero de la región del Valle Paraíba en Brasil y apoyar con ello la optimización y desarrollo del sector rural. Más recientemente, Ceballos (2013) describe con DS el comportamiento del mercado porcícola en donde se puede apreciar la dependencia del sistema a la materia prima y por lo tanto sus dificultades para aumentar la competitividad mediante el análisis de la viabilidad de inversión, presenta aspecto de producción y comercialización, instalaciones, mano de obra y alimentación.

\section{Modelado y Simulación}

El término modelo en la DS hace alusión a la representación o construcción para explicar la complejidad de una situación. Un Modelo puede ser llevado a cabo básicamente para dos propósitos, la intervención o el aprendizaje (Andrade et al., 2001). La intervención se orienta por el pronóstico que reporta la simulación, la cual permite recrear posibles escenarios de la realidad modelada al designar valores para cada una de las variables que lo representan en el modelo. La simulación, con la mediación del modelo, hace posible respuestas a preguntas referentes al fenómeno; además aporta al entendimiento sobre los mecanismos que explican el funcionamiento del sistema modelo.

\section{Ambientes de Aprendizaje}

La complejidad del modelo, en términos del número de variables, las relaciones lineales y no lineales, la diversidad de escenarios y los experimentos posibles, hace aconsejable el diseño de ambientes informáticos de experimentación y aprendizaje que faciliten la interacción simulada con el fenómeno modelado, en donde esté presente la designación de roles y medios que generan condiciones favorables para el aprendizaje (Duarte, 2003). En esta propuesta, los ambientes se estructuran en términos de un modelo de simulación, una base de datos y un componente software que facilitan la experimentación simulada.

\section{Dinámica de Sistemas (DS)}

La DS es una alternativa para dar cuenta de la complejidad dinámica de los fenómenos explicables en términos de sistemas en donde la variedad en los campos de aplicación la proponen como una metodología (Ford, 1999). La DS facilita la descripción de las relaciones entre elementos del sistema y de éste con su entorno mediante modelos que se construyen en un proceso de cobertura y complejidad creciente; fue formulada por Jay Wright Forrester del Instituto Tecnológico de Massachusetts (MIT) (Forrester, 1997). La DS se presenta como un lenguaje de representación del conocimiento según el esquema de la Figura 1 (Andrade et al, 2001), los cuales se soportan en un software, en esta propuesta se hace uso de Evolución, el cual realiza la evaluación de las ecuaciones, que escritas de la forma tradicional pueden ser poco comprensibles y no solucionables por métodos analíticos (Andrade et al. 2001). 


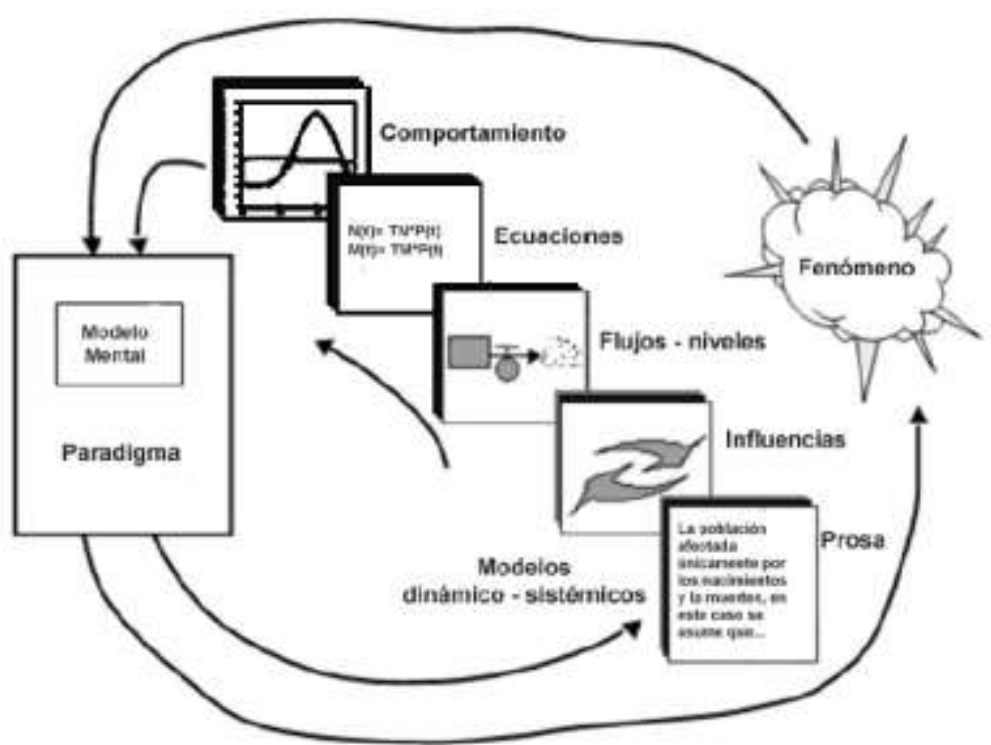

Fig. 1: Lenguajes de la DS (Andrade et al., 2001).

La Prosa es una descripción del fenómeno en lenguaje natural que permite apreciar las variables y sus relaciones. El Diagrama de Influencias es un esquema donde se integran las variables identificadas, mostrando con flechas la manera como cada una influye a las demás conformando ciclos de realimentación de refuerzo o positivos y compensadores o negativos. Los Flujos y Niveles son una representación basada en elementos propios de la DS que representan ecuaciones diferenciales lineales o no lineales, Figura 2.

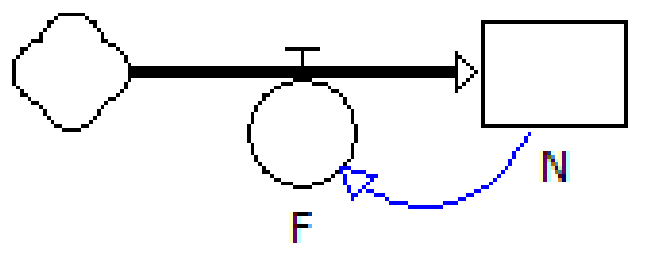

Fig. 2: Estructura Básica de Flujo-Nivel

La evaluación que hará el software en cada paso de simulación generará el comportamiento del sistema; la relación entre la variable Flujo $(\mathrm{F})$ y la variable Nivel $(\mathrm{N})$ de la figura 2, equivale a la expresión diferencial presentada a continuación y que el software asume en términos de una ecuación en la notación de Euler así:

$$
\begin{aligned}
& \frac{\mathrm{d}(\mathrm{N})}{\mathrm{dt}}=\mathrm{F}(\mathrm{t}) \\
& \mathrm{N}(\mathrm{t}+\Delta \mathrm{t})=\mathrm{N}(\mathrm{t})+\mathrm{F}(\mathrm{t})^{*} \Delta \mathrm{t}
\end{aligned}
$$

La anterior ecuación indica que $\mathrm{N}$ en el cada instante de tiempo se obtiene sumando al $\mathrm{N}$ que existía en el tiempo $t$, con el $\mathrm{F}$ (flujo) calculado en $\mathrm{t}$ y asumido constante durante el período de tiempo $\Delta \mathrm{t}$. La ecuación indica cómo evoluciona la variable de estado $\mathrm{N}$ en función del flujo $\mathrm{F}$ que determina su variación. Además de Flujos y Niveles, en un modelo de DS están presentes otros elementos y relaciones que se describen en términos de Parámetros, Variables Auxiliares y Exógenas, Retardos, Multiplicadores, Clones y Sectores (Forrester, 1997; Andrade et al., 2001; Sterman, 2000). Las Ecuaciones se hacen explícitas en el lenguaje de los flujos (lo que genera el cambio) y los niveles (lo que cambia) pero la asignación de la relación matemática de las variables involucradas se deben definir como otro lenguaje y el comportamiento es la presentación de los resultados que arrojan las simulaciones.

\section{Sistemas de Producción Agropecuario}

El sistema de producción agropecuaria, es asumido generalmente como una propiedad individual conocida como finca o empresa agroindustrial (Gómez y Barragán, 2002; Gómez et al., 2003) que contempla el subsistema productivo con animales de una misma especie como bovinos, caprinos, cunícolas o porcinos. En los aspectos determinantes de la producción agropecuaria es necesario considerar la cantidad y la 
calidad de los recursos demográficos, biofísicos, productivos y económicos, la disponibilidad y facilidad de medios de producción, las condiciones particulares del mercado de insumos y productos, entre otros. La administración de la finca requiere una visión sobre la dinámica como se relacionan e interactúan cada uno de los elementos que la conforman. La interpretación y profundización en el conocimiento a nivel de finca es necesaria para pensar y entender las complejidades del sistema (Phillips, 2003) y orientar el diseño de políticas de intervención en el mismo.

En esquemas integrales de producción, los volúmenes de datos son de tal magnitud y de tan diversa naturaleza que requieren herramientas computacionales para visualizar y manejar las interacciones entre los aspectos contemplados (Gómez, 2010); así mismo, la diversidad en la apreciación de estos esquemas integrales y la complejidad del sistema que los describe, demanda equipos interdisciplinarios para la construcción de los modelos asociados a los mismos.

\section{PROPUESTA METODOLÓGICA}

La frase "la experiencia no es lo que le sucede al hombre, sino lo que el hombre hace con lo que le sucede" de Aldous Huxley (1894-1963. Novelista, ensayista, crítico y poeta inglés); puede ser asumida para sustentar el aprendizaje con ambientes de modelado y simulación con DS, al facilitar a los usuarios: i) Recrear y apreciar el devenir mediante la simulación en diversos escenarios; ii) Reflexionar sobre los sucesos con apoyo en el conocimiento que sintetiza el modelo, aprendiendo; y iii) Construir así un nivel de experiencia que puede guía nuevas y mejores intervenciones en el fenómeno. Ambrosio (2011) manifiesta que orientar las investigaciones de las diferentes disciplinas apoyadas en el pensamiento sistémico contribuye en la comprensión de la complejidad de los fenómenos; e ilustra su afirmación con el caso de un sistema de producción lechero en donde, mediante la construcción de un modelo en el lenguaje de las influencias da a conocer aspectos que aportan en la generación, adaptación y transferencia de conocimientos científicos y tecnológicos que facilitan la toma de decisiones.

Desde la perspectiva anterior, es posible afirmar que los sistemas de información para el sector agropecuario comúnmente son limitados para promover procesos de aprendizaje y para apoyar la toma de decisiones; aunque permiten almacenar un historial de los datos demográficos, productivos y económicos, no siempre facilitan los pronósticos bajo diferentes escenarios, ni la confrontación de resultados para optimización en cada uno de los subsistemas. Una alternativa que atienda las limitaciones señaladas puede apoyarse en el modelado matemático de enfoque estructural como el de la DS. Estos modelos facilitan comprender la estructura del fenómeno modelado, el comportamiento de las variables de interés y responder a preguntas de la forma: ¿Qué podría pasar si, dado un escenario particular, realizamos $X$ o $Y$ intervención? Existen evidencias de la usabilidad de ambientes software de modelado y simulación tales como: Cazanga (2009), Ruiz y Silva (2008) y Gómez et al. (2003); que muestran la posibilidad de desarrollar competencias mediante su uso, de una manera similar a como los simuladores de vuelo apoyan el aprendizaje, permitiendo vivir situaciones posibles para los pilotos y que les aportan experiencias que fortalecen su capacidad de toma de decisiones.

El desarrollo de modelos con DS orientados a la comprensión del comportamiento de los sistemas de producción agropecuario, contemplando lo demográfico de manera integrada con lo biofísico, productivo y económico, es baja dada la complejidad de estos sistemas productivos. Este documento propone unos lineamientos guía para asumir la construcción de dichos modelos y del componente software para operar con ellos; promoviendo el aprendizaje con experiencias simuladas, a semejanza de las que se pueden presentar en la relación con el fenómeno real.

\section{Lineamientos para el Modelado de los Sistemas de Producción}

Los sistemas de producción agropecuaria, se pueden describir mediante una estructura común, definida en términos de los subsistemas demográfico, biofísico, productivo y económico relacionados entre sí constituyendo un complejo sistema dinámico, cuya estructura general incorpora la hipótesis dinámica básica que se puede apreciar en la

Fig. 3; esta hipótesis fue formulada a partir de la depuración de resultados de experiencias de investigación, desarrollo y revisión de modelos poblacionales con DS similares a los contemplados en los ambientes software SIPROB 1.0 (Gómez et al., 2003) y SIPROB 2.0 (Gómez et al., 2010), del grupo SIMON (Grupo SIMON de Investigación. 2008) y otros trabajos como el de Díaz (2008), Lellis et al., (2011) y Toledo et al, (2011).

La complejidad dinámica de los modelos está asociada a la complejidad de su estructura realimentada, de tal forma que las perturbaciones en una de las variables se transmite en los ciclos retornando sobre la variable inicial (realimentación); por ejemplo, las salidas del subsistema económico permiten nuevas 
entradas en el demográfico y, además, según los elementos contemplados para cada subsistema, es posible centrar su producción en Cría, Ceba, Leche o Doble propósito.

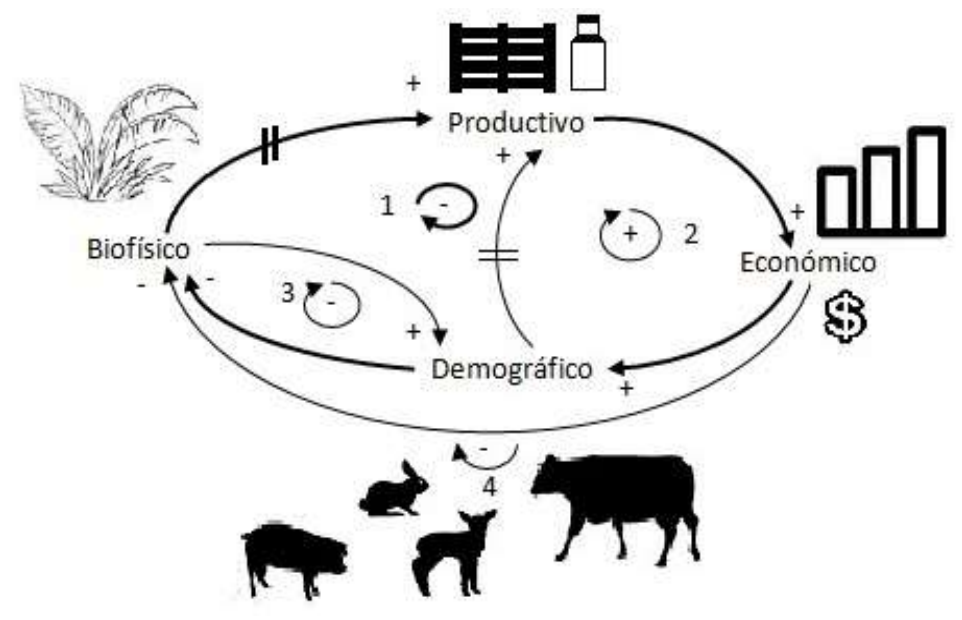

Fig. 3: Hipótesis Dinámica Básica del Sistema Productivo Agropecuario

La hipótesis dinámica básica relaciona los cuatro subsistemas endógenos que interactúan estableciendo ciclos de realimentación. Los ciclos de refuerzo o positivos (+), describen el crecimiento en función del efecto de realimentación que refuerza cualquier modificación en sus elementos; los ciclos compensadores o negativos (-), explican la tendencia a la estabilidad o búsqueda de meta por el sistema, al compensar los efectos de cambio en alguno de los elementos; de esta manera los ciclos de realimentación negativos revelan los límites en el crecimiento de cualquier sistema productivo. La dinámica resultante de la relación entre ciclos de refuerzo y ciclos compensadores, determina el devenir global del sistema modelado y, en este caso, sintetizan la hipótesis dinámica del mismo y es descrita por cuatro ciclos de realimentación, tres compensadores y uno de refuerzo y a su vez es afectada por dos retardos (identificados con dos barras paralelas sobre la relación II) que representan demoras en la manifestación de efectos, por ejemplo, del efecto resultante al modificar la raza o el tipo de pasto.

Aunque la dinámica del sistema es el resultado de la compleja integración entre los cuatro ciclos y los retardos ya señalados, es posible apreciar el papel general de cada uno de ellos en dicha dinámica: i) Los cuatro subsistemas se integran en el ciclo $1(-)$; lo cual deja ver que frente a una política de inversión económica en lo demográfico, el sistema tiende a un límite de crecimiento determinado por el carácter finito del subsistema biofísico; ii) El ciclo 2 (+) describe la relación entre lo económico, lo demográfico y lo productivo, y explica el efecto de crecimiento que es posible con la intervención en cualquiera de estos tres subsistemas; iii) El ciclo 3 (-), impone límite al crecimiento del subsistema demográfico, por su relación con lo biofísico; iv) El ciclo 4 (-), contempla la posibilidad de crecimiento del subsistema productivo frente a políticas de inversión económica en lo biofísico.

Es de señalar que la hipótesis dinámica, asumida en su conjunto, explica el crecimiento y equilibrio dinámico que puede mostrar un sistema productivo de este tipo y la apreciación global que construye un gerente al simular con estos ambientes reduce su incertidumbre en la toma de decisiones y facilita evaluar los posibles efectos de las diversas alternativas de política de gestión y/o del uso de tecnologías como de mejora de razas, inversión en equipos, recuperación de pastos, control de plagas, entre otras; lo cual no es posible cuando asume cada subsistema de manera independiente. Estos sistemas productivos, además de la estructura básica que comparten, presentan aspectos similares (Tabla 1). Similitudes que permiten que el proceso de modelado pueda iniciar con un modelo global común y posteriormente considerar las características que distinguen cada sistema productivo.

Igualmente, las características comunes conducen a similitudes en la gestión del sistema de producción y, por ende, a identificar una estructura básica para los modelos, que determina una lógica común que explica la dinámica, es decir, un isomorfismo. Por ejemplo: En todos debe desarrollarse la relación Costo-Beneficio a partir de las actividades de comercialización (compras, ventas) de los ejes productivos (carne, leche, abono, cueros). Así mismo, estas características permiten formular similitudes a un nivel de mayor profundidad y detalles, mediante la explicación estructural de la dinámica del sistema productivo en términos de un modelo básico de Flujo-Nivel como el que es presentado en la Fig. 4, en la cual se consideran los elementos de la hipótesis dinámica básica de la 
Fig. 3. La tabla 2 describe el diagrama de Flujo-Nivel de la Figura 4 para cada uno de los subsistemas, en uno de los modelos básicos propuestos, para agrupar los subsistemas de la dinámica básica.

Tabla 1: Características generales de los sistemas de producción

\begin{tabular}{|l|c|c|c|c|}
\hline Características Generales & Bovino & Porcino & Caprino & Cunícola \\
\hline Gestación (Incubación) [días] & 270 & 114 & 150 & 31 \\
\hline Crías promedio al parir & 1 & 9 & 2 & 7 a 9 \\
\hline Lactancia [días] & 270 & 21 & 100 & 56 \\
\hline Recuperación hembras [días] & 90 & 14 & 50 a 60 & 17 \\
\hline \# Ideal de partos por año & 1 & 2.7 & 1.7 & 5 a 7 \\
\hline \# Promedio de crías por año & 1 & 24 & 1 & 35 \\
\hline
\end{tabular}

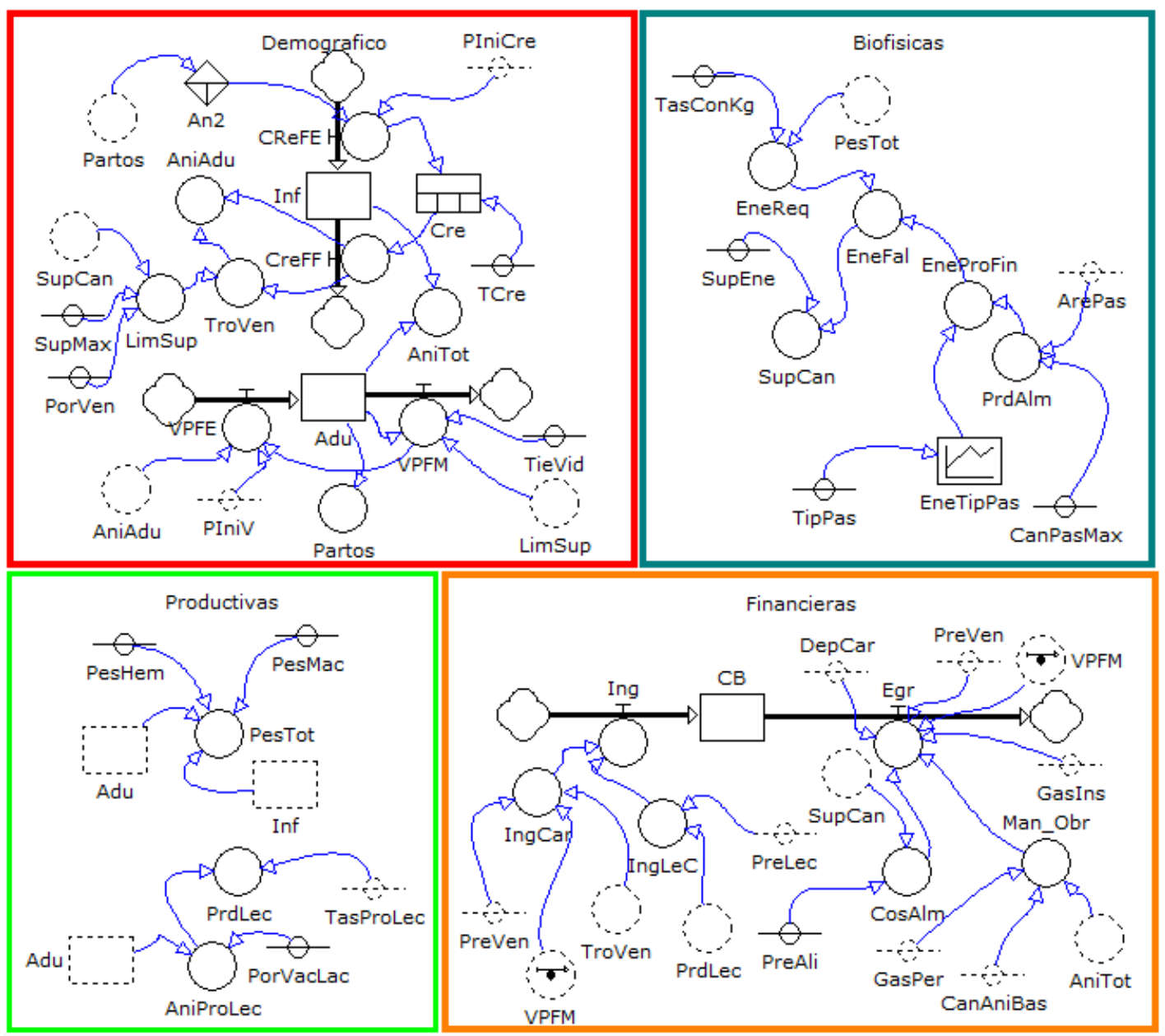

Fig. 4. Modelo resumido de la hipótesis de la dinámica básica en Flujos y Niveles

Tabla 2 Descripción del diagrama de Flujo-Nivel de la hipótesis de la dinámica básica.

\begin{tabular}{|l|l|}
\hline Subsistema & Descripción \\
\hline Demográfico & $\begin{array}{l}\text { Representa los grupos etarios en Niveles. Para el caso presentado, Infantes (Inf) y Adultos (Adu); los } \\
\text { cuales varían en función de los flujos de crecimiento, que son afectados por la Población Inicial } \\
\text { (PIniCre), los Partos y los indicadores de crecimiento otorgado por el suplemento (SupCan), éste se } \\
\text { encuentra en líneas punteadas por que corresponde al subsistema biofísico. El flujo de salida } \\
\text { representa que después de un tiempo de crecimiento (TCre), los animales machos son vendidos y } \\
\text { esta acción se manifestará en el subsistema económico. }\end{array}$ \\
\hline Biofísico & $\begin{array}{l}\text { Permite representar los factores alimenticios, es este subsistema es estimada la energía faltante } \\
\text { (EneFal) para alimentar los animales a partir de la cantidad de energía requerida (EneReq) y la } \\
\text { cantidad de energía producida en la finca (eneProFin) a partir del área cultivada (ArePas). El peso } \\
\text { en animales (PesTot) proviene del subsistema productivo y por ello se encuentra punteado. }\end{array}$ \\
\hline Productivo & $\begin{array}{l}\text { A partir de los Infantes (Inf) y Adultos (Adu) y el promedio de sus pesos es calculado el Peso Total y } \\
\text { por indicadores de producción de Leche (TasProLec) y el porcentaje de vacas lactantes (PorVacLac) } \\
\text { de la hembras adultas (Adu) es estimada la producción de Leche (prdLec) que beneficiará el }\end{array}$ \\
\hline
\end{tabular}




\begin{tabular}{|l|l|}
\hline & subsistema Económico. \\
\hline Económico & $\begin{array}{l}\text { Se concentra en estimar la relación costo-beneficio (CB) a partir de los Ingresos (Ing) (venta de } \\
\text { animales y leche) y Egresos (Egr) (Suplemento, Mano de Obra y Gasto de las instalaciones). }\end{array}$ \\
\hline
\end{tabular}

\section{Subsistema Demográfico}

El modelado del subsistema demográfico contempla los estados de cada uno de los grupos etarios y las posibles acciones en este elemento, lo cual determina el nivel de complejidad de la representación en el lenguaje de Flujo - Nivel, en donde para cada grupo etario (Niveles) se pueden presentar Entradas 0 Salidas (flujos). Las Entradas se dan por compras, por la llegada de animales de otro grupo etario o por los partos. Las Salidas se dan por los descartes, las ventas, las muertes o por cumplir el ciclo al que pertenece.

La figura 5 especifica el subsistema etario general en el lenguaje de influencias, identificando la estructura de realimentación para los casos de bovinos, porcinos, caprinos y cunícolas; estructuras que son leídas en la en la Tabla 3.
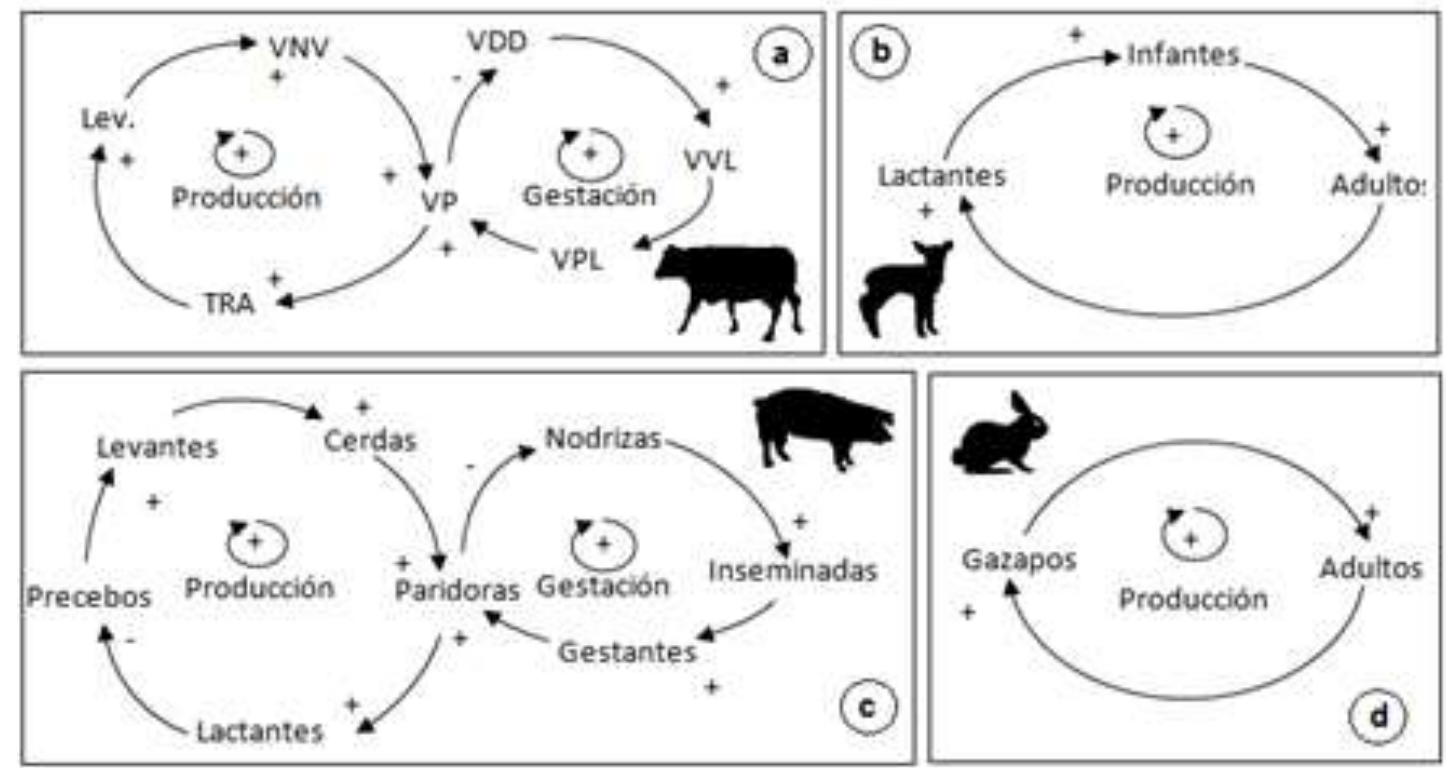

Fig. 5: Diagrama de Influencias del Subsistema Etario: Caso Bovino, Porcino, Caprino y Cunícola

Tabla 3. Ciclos de los Diagramas de Influencias para cada subsistema etario

\begin{tabular}{|c|l|}
\hline Sistema & \multicolumn{1}{|c|}{ Descripción del Ciclo } \\
\hline Bovino & $\begin{array}{l}\text { En el primer ciclo que se presenta se puede apreciar que a mayor cantidad de Terneras (TRA), mayor } \\
\text { será la cantidad de Levantes (LEV); a mayor cantidad de LEV, mayor será la cantidad de Vacas Nuevo } \\
\text { Vientre (VNV); a mayor cantidad de VNV, mayor será la cantidad de Vacas preñadas (VP); a más VP, más } \\
\text { Terneras (TRA); completando el primer ciclo de refuerzo, en el otro ciclo, también de refuerzo, se tiene } \\
\text { que a mayor cantidad de VP, mayor es la cantidad de Vacas en Días de Descanso (VDD); a mayor } \\
\text { cantidad de VDD, mayor será la cantidad de Vacas Vacías Lactantes (VVL); a mayor cantidad de VVL, } \\
\text { mayor será la cantidad de Vacas Preñadas (VPL); a mayor cantidad de VPL, mayor será la cantidad de } \\
\text { VP. }\end{array}$ \\
\hline Porcino & $\begin{array}{l}\text { En el primer ciclo que se presenta se puede apreciar que a más Lechones Lactantes, mas Precebos; a } \\
\text { más Precebos, mas Cerdas en Levante; a más Cerdas en Levante, más Cerdas, a más Cerdas, más } \\
\text { Paridoras; a mas Paridoras, más Lactantes completando el primer ciclo de refuerzo, en el otro ciclo, } \\
\text { también de refuerzo, se tiene que a más Paridoras, más Nodrizas; a más Nodrizas, más Hembras en } \\
\text { Inseminación; a más Hembras en Inseminación, más Cerdas en Gestación; a más Cerdas en Gestación; } \\
\text { más Paridoras. }\end{array}$ \\
\hline Caprino & $\begin{array}{l}\text { En el ciclo de refuerzo que se presenta se puede apreciar que a más Lactantes, más Infantes; a más } \\
\text { Infantes, más Adultos; a más Adultos, más Lactantes. }\end{array}$ \\
\hline Cunícola & $\begin{array}{l}\text { En el ciclo de refuerzo que se presenta se puede apreciar que a más Gazapos, más Adultos; a más } \\
\text { Adultos, más Gazapos. }\end{array}$ \\
\hline
\end{tabular}

Al diagrama de influencias de los subsistemas demográficos de la Fig. 5, se deben agregar algunos elementos como las tasas de mortalidad, natalidad y crecimiento, las muertes y los partos para luego formular el modelo en términos del lenguaje de Flujos y Niveles como se aprecia en la Fig. 6. 

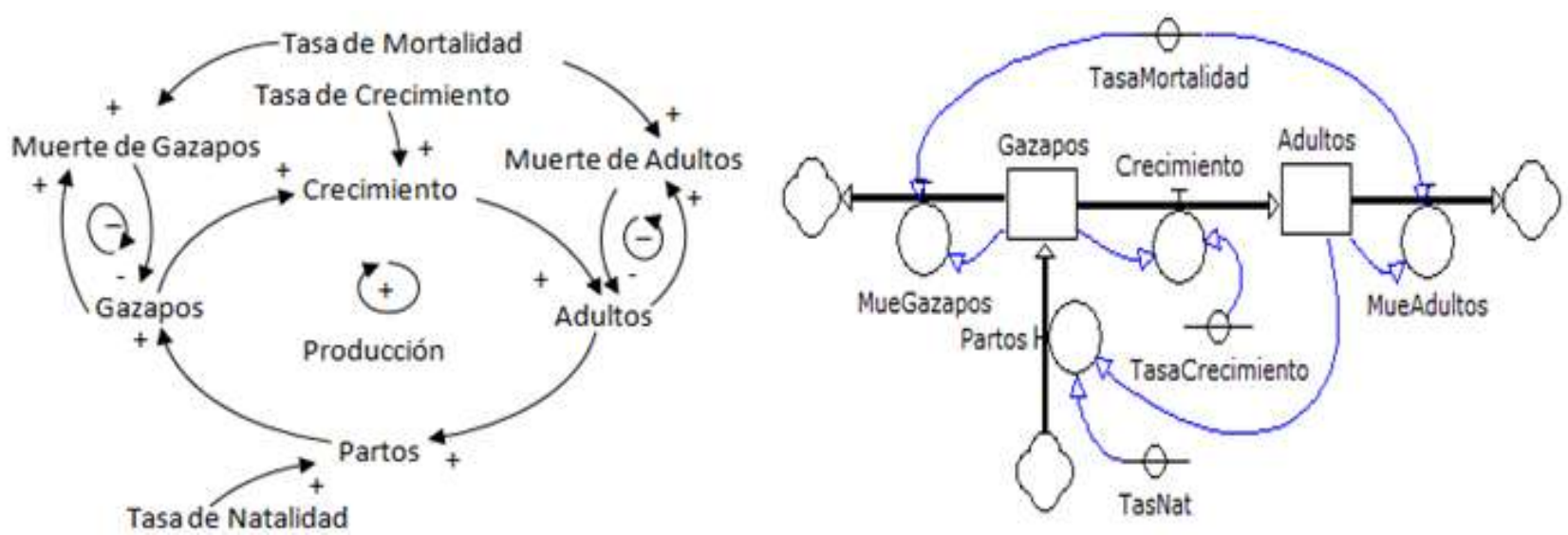

Fig. 6: Diagrama de Influencias y de Flujos-Niveles del subsistema demográfico: Caso Cunícola

A partir del diagrama de flujo-nivel y teniendo presente el significado de cada parámetro y variable, se formula, en términos de ecuaciones explícitamente definidas, el modelo matemático con el cual es posible simular la dinámica del subsistema etario bajo diferentes escenarios. Para ilustrar este proceso se recrea el sistema cunícola, para el cual las ecuaciones, en la forma de Euler, con las cuales opera la simulación y que corresponden con el diagrama Flujo-Nivel de la Fig. 6, son:

Gazapos $(\mathrm{t}+\Delta \mathrm{t})=$ Gazapos $(\mathrm{t})+\left(\right.$ Partos $\left.(\mathrm{t}){ }^{*} \Delta \mathrm{t}\right)-\left(\right.$ Crecimiento $\left.(\mathrm{t}){ }^{*} \Delta \mathrm{t}\right)-($ MuerteGazapos $\left.(\mathrm{t})){ }^{*} \Delta \mathrm{t}\right)$

Adultos $(\mathrm{t}+\Delta \mathrm{t})=$ Adultos $(\mathrm{t})-\left(\right.$ Crecimiento $\left.(\mathrm{t}){ }^{*} \Delta \mathrm{t}\right)-($ MuerteGazapos $\left.(\mathrm{t})){ }^{*} \Delta \mathrm{t}\right)$

Partos $(\mathrm{t})=$ Adultos $(\mathrm{t}){ }^{*}$ TasaNatalidad

Crecimiento $(\mathrm{t})=$ Gazapos $(\mathrm{t})$ * TasaCrecimiento

MuerteGazapos $(\mathrm{t})=$ Gazapos $(\mathrm{t}){ }^{*}$ TasaMortalidad

MuerteAdultos $(\mathrm{t})=$ Adultos $(\mathrm{t}){ }^{*}$ TasaMortalidad

Con un modelo en ecuaciones y en un escenario asociado, es posible, mediante la simulación, apreciar la dinámica del sistema, en términos del lenguaje del comportamiento. Por ejemplo, con un prototipo de modelo para producción bovina coherente con la figura $5^{\underline{a}}$ es presentado en la Figura 7, el resultado de simular las variables demográficas, en donde se aprecia que: i) En la primera semana de simulación solo hay diez Vacas Nuevo Vientre (VNV); ii) En la quinta semana, nueve de esas VNV han sido preñadas (VP); iii) En la semana treinta y nueve, ya se han presentado ocho partos y que según la tasa de natalidad contemplada en el escenario, hay dos VP, siete vacas en días de descanso (VDD), y los partos produjeron tres terneras (TRO[2]). En la semana cuarenta, ya se presentaron los partos y la mitad fueron hembras; iv) En la semana cincuenta y uno se ha terminado el periodo de descanso de cinco vacas que ahora se encuentran en el grupo etario de vacas vacías lactantes (VVL); y v) En la semana cincuenta y tres, hay tres VVL, tres VPL y tres vacas vacías (VV), muy cerca de que el ciclo vuelva a comenzar.

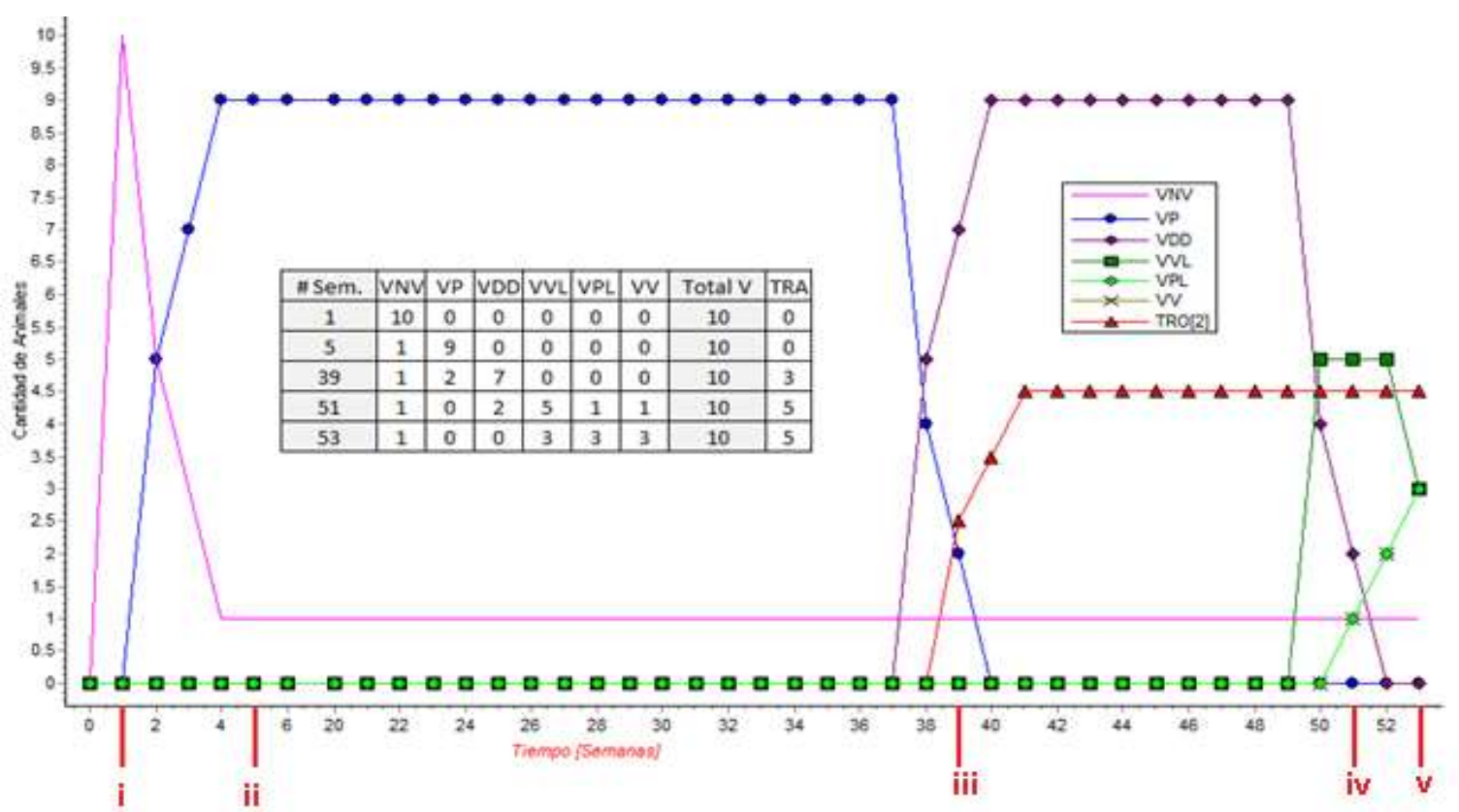

Fig. 7: Comportamiento del subsistema Etario: Caso Bovinos 


\section{Subsistema Biofísico}

Contempla los aspectos que determinan la capacidad productiva de la finca como, por ejemplo, la alimentación requerida según la cantidad de animales en el sistema de producción, el área de la finca, el tipo de pasto y el suplemento; con estos elementos el modelo determina la capacidad de carga de la finca y por ende las compras o descartes que se deben realizar.

\section{Subsistema Productivo}

Incluye los factores a los cuales los administradores de las fincas deben prestar mayor atención, como las tasas de producción de leche, carne, lana y pasto; asumidas en este subsistema como elementos exógenos constitutivos de los diferentes escenarios.

\section{Subsistema Económico}

Todo administrador presta atención especial a este componente, "la punta del iceberg" (Andrade et al., 2001) de la dinámica del sistema productivo. Describe la estructura que integra las variables que permiten calcular los ingresos y los egresos a partir de las variables productivas, biofísicas y demográficas.

\section{Integración de los Componentes}

Un proceso de modelado por prototipos de complejidad y cobertura creciente (Andrade et al., 2001), se desarrolla guiado por la hipótesis dinámica básica (

Fig. 3) y, en este caso, a partir de las estructuras presentadas para el subsistema demográfico (Fig. 5) e integrando los demás. En la medida que se desarrolla el modelo se incrementa el número de elementos, las relaciones, la complejidad estructural y matemática y la cobertura del sistema productivo.

\section{Componente Software para la Simulación}

Los ambientes de aprendizaje que contemplan modelos complejos como los de producción agropecuaria requieren además de los modelos, un componente software con una base de datos que facilite operar con el modelo para realizar diversas simulaciones y experimentos con diferentes propósitos.

El componente software, desarrollado para ilustrar estos lineamientos fue denominado AgroDiSi (Software para la simulación de modelos Agropecuarios con Dinámica de Sistemas) y presenta alternativas de uso que brindan a cada uno de los tipos de usuario las posibilidades de interactuar con los modelos. Un ejemplo de las funcionalidades básicas se presenta en el diagrama de casos de uso de la Fig. 8.

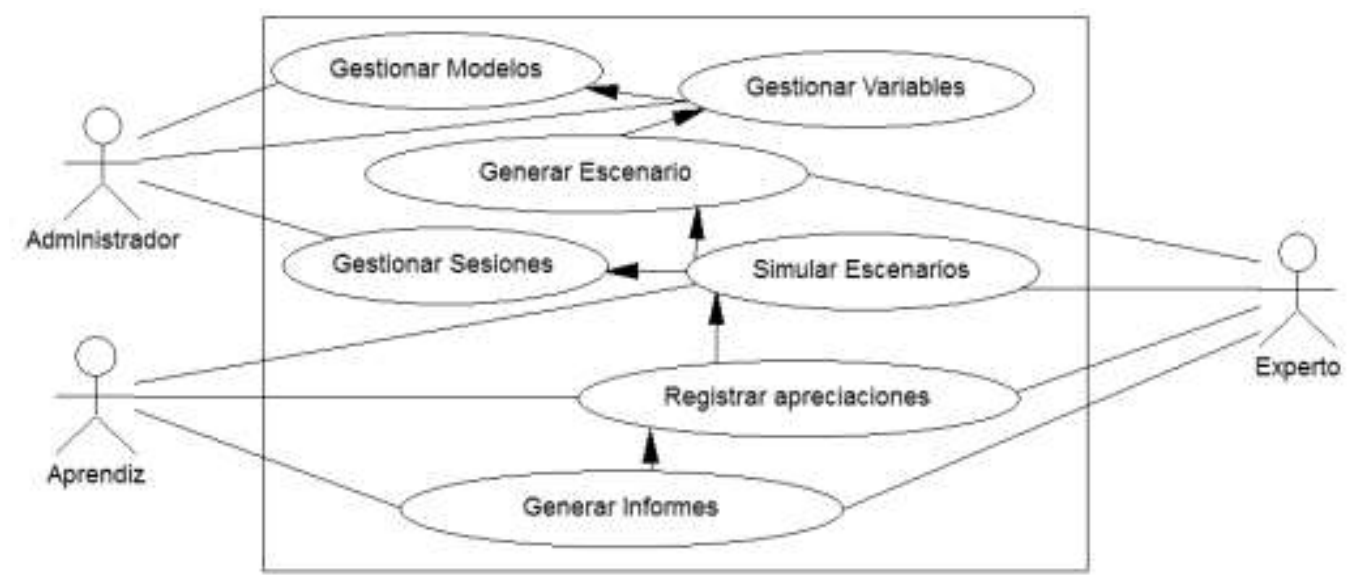

Fig. 8: Diagrama de Casos de Uso de AgroDiSi.

En la base de datos se almacena información de los usuarios, los modelos, las variables que lo componen, las modificaciones de estas para los diferentes escenarios, los comportamientos simulados y las conclusiones que registran los usuarios. La interacción entre los componentes de AgroDiSi facilita el aprendizaje y la toma de decisiones, al recrear la dinámica del fenómeno explicado mediante la simulación de múltiples experimentos. Una de las pantallas de AgroDiSi es presentada en la Fig. 9, en la cual se aprecia parte del análisis de resultados entre diferentes escenarios en donde se pueden obtener respuestas a preguntas de la forma “¿Qué podría pasar si...?”. Además AgroDiSi permite la interacción para tres perfiles: Aprendiz, Experto y Administrador; cuyo alcance y descripción es: 
i) Al Experto le brinda facilidades para la experimentación con los modelos evaluando alternativas para la toma de decisiones mediante el análisis de sensibilidad por variación de parámetros o por la comparación del comportamiento resultante en diferentes escenarios, por ejemplo, con diferentes actividades orientadas a mejorar la administración. De tal forma que cada experimento permite disminuir la incertidumbre en las decisiones para intervenir de manera virtual en el proceso productivo.

ii) Al Aprendiz se le facilita su formación, al presentarle la explicación en términos del modelo, con el cual puede recrear situaciones bajo diversos escenarios y apreciar las respuestas ante posibles cambios en las variables o parámetros para el modelo y variables que se le asignen.

iii) Al Administrador le permite la gestión de los modelos y las variables, es él quien debe responder por la información que se agregue, por ejemplo: los valores mínimos y máximos de cada variable o la cantidad de simulaciones que debe realizar el aprendiz para cada modelo antes de avanzar a modelos de mayor cobertura y/o complejidad

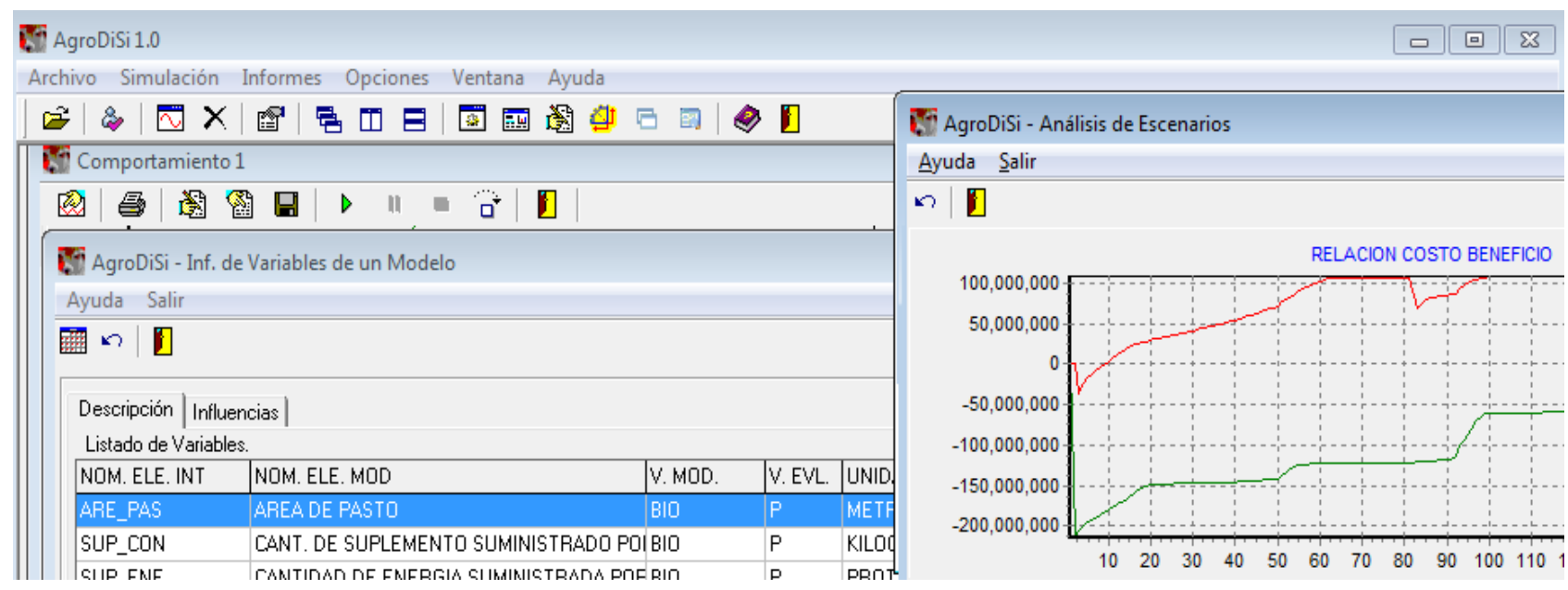

Fig. 9: Imagen de la interface de AgroDiSi

\section{CONCLUSIONES}

La triada de: lineamientos metodológicos para el modelado de sistemas productivos pecuarios (en este caso soportada en el modelamiento con el lenguaje de la Dinámica de Sistemas), junto con un software especializado para modelamiento con DS y un ambiente informático para el uso del modelo; se aprecia como útil y conveniente para aprendices e investigadores que en grupos interdisciplinarios asumen el reto de abordar la complejidad Dinámico Sistémica de estos sistemas productivos, en un proceso de aprendizaje y construcción de explicaciones científicas, mediante la matemática no lineal y demás herramientas de representación del conocimiento, que la tecnología actual coloca al alcance de especialistas de cualquier área.

Así mismo, las experiencias señaladas de uso de la Dinámica de Sistemas para la comprensión de la complejidad dinámica de los sistemas de producción pecuaria, dan sentido a los lineamientos metodológicos aquí esbozados, para el modelamiento con DS de estos sistemas productivo, tanto en la fase de construcción del modelo conceptual (representado principalmente en términos de diagramas de influencias realimentados), como del modelo computacional o modelo matemático de simulación.

Además, estos planteamientos constituyen un llamado a fortalecer con la tecnología, el Pensamiento Sistémico y la Dinámica de Sistemas, los espacios de práctica y encuentro interdisciplinario y multidisciplinario que constituyen comunidades científicas como la sociedad internacional de DS (the System Dynamics Society), el capítulo Latinoamericano, la comunidad Colombiana, la Brasilera, la Argentina y demás capítulos regionales y comunidades nacionales que en el mundo promueven la DS.

\section{AGRADECIMIENTOS}

Los autores agradecen a la Universidad Pontificia Bolivariana y a la Universidad Industrial de Santander en Bucaramanga, Colombia, por brindar las condiciones necesarias para la ejecución de las labores académicas y de investigación que permitieron esta publicación, a Don Eliécer Gómez, propietario de una finca ganadera en Betulia (Santander-Colombia) donde se corroboraron planteamientos teóricos y al Instituto de Proyección Regional y Educación a Distancia (IPRED) de la Universidad Industrial de Santander que generó los espacios para realizar las pruebas de AgroDiSi. 


\section{REFERENCIAS}

Ambrósio, L. A. Introdução da Dinâmica de Sistemas em Instituições de Pesquisa: Um estudo de caso. Memorias del IX Congreso Latinoamericano de Dinámica de Sistemas y II Congreso Brasilero de Dinámica de Sistemas, Brasil (2011)

Andrade H, Dyner I, Espinosa A, López H, Sotaquirá R.; Pensamiento Sistémico: Diversidad en búsqueda de Unidad. Ediciones Universidad Industrial de Santander (2001)

Andrade H, Lince E, Mercado A, Monsalve A. Evolución: herramienta software para modelado y simulación con Dinámica de Sistemas. Revista de Dinámica de Sistemas Vol. 5 Núm. 1. (2011)

Cazanga R.; Modelos de simulación en agricultura; Acceso 3 de Julio (2011). http://www.ciren.cl/datos/archivo/pdf/modsimulacionagricultura.pdf (2009)

Ceballos Y., Maribel Uribe M. y Sánchez G. Modelo de Dinámica de Sistemas para la Predicción del Comportamiento del Mercado Porcícola. Inf. Tecnol. 24(4), 117-124 (2013)

Checkland P.; Systems Thinking, Systems Practice. West Sussex. John Wyley \& Sons Ltd (1999)

Corporación Colombia Internacional (CCl), y Ministerio de Agricultura y Desarrollo Rural de Colombia (MADR). Caracterización de la producción de leche en Colombia. $1^{\circ}$ ed. Bogotá. (2010)

Díaz R.; Sistema cerdos-pastos-leche: Modelo de análisis económico y ambiental. CORPOICA, Colombia. Acceso 18 de Marzo (2014). http://www.condesan.org/publicacion/bgris/colombia/colombia5.html (2008)

Duarte J.; Ambientes de aprendizaje: Una aproximación conceptual; Acceso 5 de Julio (2011). http://www.rieoei.org/rec_dist1.htm (2003)

Ford, A. Modelling the environment. An Introduction to system dynamics. Models of environmental systems. Island Press. Washington DC (1999)

Forrester J.; Road Maps: A guide to learning system dynamics. Boston: System Dynamic Group, Sloan School of Management, MIT, The United States of America (1997)

Gómez UE.; Lineamientos metodológicos para la construcción de modelos agro-industriales identificables en términos de dinámicas poblacionales basados en el pensamiento sistémico y la dinámica de sistemas, Tesis de Maestría. Universidad Industrial de Santander, Bucaramanga (2010)

Gómez UE, Andrade HH.; Ambiente software para el aprendizaje de sistemas de producción de ganadería bovina, mediante simulación con dinámica de sistemas y un enfoque sistémico. SIPROB 2.0. Memorias del Sexto Congreso Latinoamericano de Dinámica de Sistemas, Chile (2010)

Gómez UE, Barragán OA, Andrade HH.; Modelo de Simulación para la Investigación Integral de Sistemas de Producción de Ganadería Bovina. Un Enfoque Sistémico. SIPROB 1.0. Memorias del Primer Congreso Latinoamericano de Dinámica de Sistemas, México (2003)

Grupo SIMON de Investigación. Grupo de Investigación en Modelado y Simulación; Acceso 18 de Octubre (2014). http://simon.uis.edu.co (2008)

Lellis Vieira, A C.G, Nicholson, Charles Frederick, Hobbs, Peter. Rural Development and System Dynamics: An Ex Ante Evaluation of Conservation Agriculture to improve Dairy Farming Systems in the Brazilian Paraíba Do Sul Valley. Memorias del IX Congreso Latinoamericano de Dinámica de Sistemas y II Congreso Brasilero de Dinámica de Sistemas, Brasil (2011)

Ospina-Pineda, D. P.; ¿Qué es un ambiente virtual de aprendizaje? Acceso 2 Noviembre (2014). http://aprendeenlinea.udea.edu.co/banco/html/ambiente_virtual_de_aprendizaje (2011)

Phillips C.; Principios de Producción Bovina. Traducido por: George, David. Edición en Lengua Española. Editorial Acribia S.A., Zaragoza, España (2003)

Sterman J.; Business Dynamics: Systems Thinking and Modeling for a Complex World. Irwin McGraw-Hill. The United States of America (2000)

Toledo L. M., Paranhos da Costa M.J.R., Ambrósio L. A; Modelo Computacional da Dinâmica do Comportamento DiádicoVaca-Bezerro. Memorias del Noveno Congreso Latinoamericano de Dinámica de Sistemas. Brasil (2011) 
